\title{
Preoperative measurement of serum CA-125 levels: is it useful in the risk assessment of low volume lymph node disease in cervical cancer?
}

\author{
Marcin Sniadecki ${ }^{1, *}$, Szymon Wojtylak ${ }^{2}$, Ewa Wycinka ${ }^{3}$, \\ Sambor Sawicki ${ }^{1}$, Juliusz Kobierski ${ }^{1}$, Marcin Liro ${ }^{1}$, Dariusz Grzegorz Wydra ${ }^{1}$ \\ IDepartment of Gynecology, Gynecologic Oncology and Gynecologic Endocrinology, \\ Medical University of Gdansk, Poland, \\ ${ }^{2}$ Department of Patomorphology, Medical University of Gdansk, Poland, \\ ${ }^{3}$ Department of Statistics, Faculty of Management, University of Gdansk, Poland
}

\begin{abstract}
BACKGROUND:

Elevated serum cancer antigen 125 (CA-125) is observed in some cervical cancers (CCs). Is the correlation of CA-125 with the presence of nodal events useful in predicting early metastasis to the lymph nodes?

METHODS:

The study included 45 patients with CC FIGO (2009) stages IA1-IIA1 and known preoperative CA-125 concentration, surgery treated (05.2011-05.2014). Investigated pretreatment: age (pre-, postmenopausal), histological type, grade, confounding factors - prior cone biopsy, ovarian cyst, endometriosis, liver or colon pathology, concomitant malignancy. LN metastases (LNM) were defined as macro (MAC, >2mm) and/or micrometastases (mic, $0.2-2 \mathrm{~mm}$ ), and LVLND as mic or/and ITC (single CC cells clusters) in LNs. Ultrastaging of all LNs (sentinel and non-sentinel, $4 \mu \mathrm{m}$ thick slices $/ 150 \mu \mathrm{m}$ intervals) was performed with hematoxylin and eosin staining and with immunohistochemistry (IHC-AE1/AE3 cytokeratin antibodies). Non-parametrical analysis and receiver operating curve analysis were used to determine correlation between CA-125 and LNM including LVLND.

RESULTS:

The median age was 55 (23-71). 806 LNs were extracted. LNM was found in 12, LVLND in 6 patients. LNM but not $L V L N D$ was correlated with higher grade $(G 2-G 3, p<0.05)$. LVLND was positively correlated with premenopausal age $(p<0.05)$ but not with tumor histology or grade. Liver disease only was found to influence CA-125 levels ( $p=0.064)$. There were no differences within CA-125 concentration among LVLND, LNM, and node-negative patients groups, however a trend was found between higher CA-125 and lower LVLND risk. CONCLUSIONS:

Elevated levels of CA-125 may be less likely due to LVLND than to LN positivity. Grade is an important feature in prediction of LNM but not LVLND. CA-125 level was found to be not predictive of LNM nor LVLND, as confirmed by ultrastaging.

Keywords: tumor biomarkers;CA-125; cervical cancer;lymph nodes metastases; ultrastaging.
\end{abstract}

Received: 30 ${ }^{\text {th }}$ December 2016; Accepted: $7^{\text {th }}$ April 2017; Published: $18^{\text {th }}$ April 2017

* Corresponding author: Marcin Sniadecki, Department of Gynecology, Gynecologic Oncology and Gynecologic Endocrinology, Medical University of Gdansk, Poland, e-mail: marcinsniadecki@gumed.edu.pl 


\section{Introduction}

Cervical cancer (CC) burden is inversely proportional to medical resources (expressed by human-development index - HDI) being the main neoplasm, together with breast cancer, among women worldwide [1]. In medium-HDI countries, such as Poland and other Middle-East European countries, $\mathrm{CC}$ is a common malignant tumor of the female genital tract $[1,2]$.

Cancer antigen 125 (CA-125) is a mucinlike glycoprotein being a marker of tumor cells differentiation. Its serum concentration correlates with the mass of living tumor cells [3,4]. The reference value for suspicion of epithelial ovarian cancer (EOC) is $<35 \mathrm{U} / \mathrm{ml}$. Broad (and nonspecific) application of CA-125 is reflected in identification of EOC, and positive lymph nodes (LNs) in endometrial cancer [5]. The increase in CA-125 level after completed treatment is an important marker for recurrence of EOC [6]. It is detected in the exo- and endocervix in $\mathrm{CC}$ [7]. Elevated CA-125 was reported in $43-75 \%$ of women with adenocarcinoma and in 19-26\% of women with squamous cell carcinoma (SCC) of the cervix [8-10]. Moreover, some studies have shown that the complementary evaluation covering CA-125 and SCC antigen (SCC-Ag) in serum is one of the prognostic factors related to the number of positive LNs and survival in SCC [11-13]. In addition, combined evaluation of CA-125 and CA 15-3 can attest to the extremely poor prognosis [14]. Some researchers postulate the measuring of SCC-Ag to be unreliable, and it is not as accessible and popular as CA-125 [14]. There are studies that show that the isolated CA125 biomarker is also suitable for monitoring the course of $\mathrm{CC}$, however, it refers rather to adenocarcinoma [15-17].

There is evidence indicating a correlation of CA-125 with both adenocarcinoma and SCC. Previous studies of the latter histological type have focused on finding a correlation of preoperative levels of CA-125 with tumor size, grade, depth of invasion, infiltration of the lymphvascular spaces and extrauterine spread of disease, including invasion of the parametrium [10-18]. However, available literature does not discuss CA-125 reference test results regarding low volume lymph node disease (LVLND, consisting of micrometastases, MICs, and isolated tumor cells, ITC) in SCC. It was found that in the group of early carcinomas (IA1-IB1 by the Fédération Internationale de Gynécologie et d'Obstétrique [FIGO]) and locally advanced (FIGO stages IB2-IIB), the incidence of lymph node metastasis is $4-15 \%$ and 12 $27 \%$, respectively $[19,20,21]$. The question is whether CA-125 biomarker may be used for determination of MICs and ITC in patients with $\mathrm{CC}$ diagnosed before surgery.

\section{Material and methods}

Figure 1 depicts a process of selecting consecutive patients from the initial group of 122 in the period between January 2010 and May 2014 in the tertiary clinical care unit (department of gynecologic oncology, medical university). Inclusion criteria were: age $\geq 18$ years-old, planned lymphadenectomy, ultrastaging performed. Exclusion criteria were: age $<18$, no lymphadenectomy performed or no ultrastaging. The final material of this study consisted of 45 consecutive patients with CC in IA1-IIA2 stages (FIGO 2009) [21], all of them underwent CA-125 assessment during preoperative hospitalization. The material was venous blood collected with vacuum tubes (Vacutainer ${ }^{\circledR}$ SST II Advance tube, Becton, Dickinson \& Co., NJ, USA; http://www.bd.com/vacutainer/). CA-125 was designated by immunochemiluminescence technique with Architect i2000SR immunoassay analyzer (Abbott Diagnostics, Lake Forrest, IL, USA; https://www.abbottdiagnostics.com/ en-us/products/ARCHITECT-i2000SR.html). 


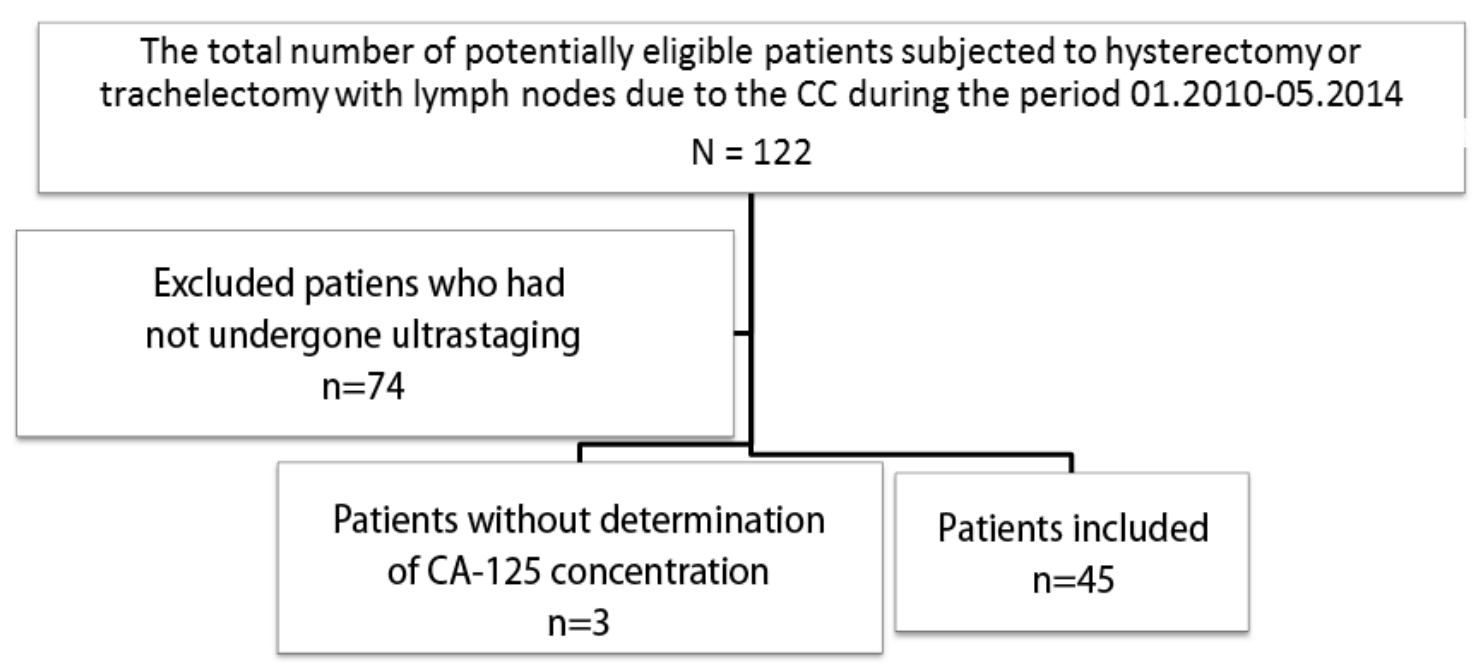

Figure 1. Selection process for eligible patients.

All patients underwent surgery adapted to the clinical stage of tumor by means of open surgery or laparoscopy. All resected LNs (806, range 3-53, mean 17.9/patient) were subjected to histopathological examination which was cutting of the specimen into 4 micrometer slides every 150 micrometers (Leica SM2010 R, Leica Biosystems, Nussloch, Germany;http:// www.leicabiosystems.com/routine-specialstaining/slidingmicrotomes/details/ product/ leica-sm2010-r/). The two sections from each level were placed on silanized slides and stained with hematoxylin and eosin (H\&E) the first one, and the second one with AE1/ AE3 clone of primary antibodies reacting with cytokeratins, by immunohistochemical (IHC) method (EnVision FLEX monoclonal Mouse Anti-Human Cytokeratin clone AE1/AE3 readyto-use catalogue nr IS053; Dako, CA, USA; http://www.dako.com/pl/ar38/psg38720000/ baseproducts.htm). Staining was performed with an automated system (AutostainerLink 48, Dako, CA, USA; http://www.dako.com/pl/index/ products/featured-solutions/autostainer-link-48.
htm).The antibodies were labeled squamous epithelium of tumors of epithelial origin. The staining reaction is positive by low as well as high expression structures. The lymph nodes (stained with HE and IHC) were evaluated using Olympus BX40 microscope (Tokyo, Japan) by a single experienced pathologist (SW). Tumor deposits were measured with built-in micrometer. The greatest dimension of each metastasis was recorded. Metastatic disease(LNM) was defined as the presence of one or more macrometastates (MAC[s]) or/and one or more micrometastates (MIC[s]), diagnosed with H\&E or/and IHC (N1 according to Tumor-Nodes-Metastases, Union Internationale Contre le Cancer/Global Cancer Control, UICC staging system). Low volume LNs disease (LVLND) was defined as the presence of MICs or/and isolated tumor cells (ITC). MAC was defined as neoplastic lesions $>2 \mathrm{~mm}$ in lymph nodes, MIC was defined as tumor deposits of $>0.2 \mathrm{~mm}$ but $\leq 2 \mathrm{~mm}$ in the LNs [22,23]. Isolated tumor cells were defined as confluent clusters of tumor cells with diameters equal to $0.2 \mathrm{~mm}$ or smaller, or single 
cells [23].An example of IHC staining is shown in supplemental material S1 presenting a lymph node with a MIC of about $1.6 \mathrm{~mm}$ in greatest dimension.

\section{Statistical analysis}

The analysis took into account variables which could affect CA-125 outcomes, like previous conization or trachelectomy, ovarian cyst (confirmed by histology or clinically - when the surgery was related to the uterus only), and comorbidities, such as bowel disease, coexistence of another cancer, liver disease and endometriosis.

Distribution of CA-125 differed from the normal distribution $(p<0.01$, KolmogorovSmirnov test with Lilliefors correction), therefore statistical analysis was performed using nonparametric methods. Mann-Whitney test (M-W) was employed to assess the relationship between CA-125 and dichotomous variables, however, the dependence of CA125 on multipartite variables was tested using nonparametric Kruskal-Wallis (K-W) ANOVA. What is more, we assumed that CA-125 is a metastasis predictor, therefore we established a measure of discrimination and calibration: ROC (Receiver Operating Characteristic) curve, the area under the ROC curve, and the Pearson chisquare statistics, respectively.

\section{Results}

The mean age of the patients was 51 (SD 13.47, range $23-71$, median 55 years). The average concentration of CA-125 in the test group was $20.9 \mathrm{U} / \mathrm{mL}$ (SD 23.018, range $4.4-$ 137.5, median 14.1). LNM was detected in 12 cases, including 6 with LVLND. Table 1 presents the number of individual variants of analyzed variables and the level of statistical significance for relation with CA-125. The analysis showed that excepting prior cervix surgery, the test group of patients was not significantly burdened by coexisting factors. LVLND but not LNM was positively correlated with premenopausal age (chisquare test, $p=0.0285$ ). The relationship between age and LNs status is shown in Table 2.

There was no statistically significant relationship between CA-125 and histological $\mathrm{CC}$ types (M-W test, $\mathrm{p}=0.907)$. There was also no statistically significant correlation of CA-125 concentration with the severity of the disease according to FIGO (K-W test, $\mathrm{p}=0.467$ ).

There was found no statistically significant correlation between CA-125 value and LNM (M-W test, $p=0,2867)$. No significant variation of ROC curve from a reference line was recorded, in addition it is repeatedly crossed (AUC 0.606; 95\% CI 0.421-0.791, $\mathrm{p}=0.2601$ ) (Figure 2). For the cut-off point $35 \mathrm{U} / \mathrm{mL}$ we obtained accuracy $=0.667$, sensitivity $=0.083$, specificity $=0.879$ of predicting LNM based on CA-125 concentration. There was no statistically significant correlation between CA-125 concentration and grade (K-W test, $\mathrm{p}=0.1692$ ), despite the fact that it was demonstrated that grade affects the risk of metastases to the LNs (chi square $p<0.04$ ). No relationship between disrupting factors and the presence of LNM for any of listed factors was found.

There was no statistically significant relationship between CA-125 value and MIC (M-W test, $\mathrm{p}=0.873$ ). The ROC curve repeatedly crosses the reference line (AUC $0.527 \quad$ (95\% CI $0.24-0.815, p=0.851$ ) (Figure 3). For the cut-off point $35 \mathrm{U} / \mathrm{mL}$ we obtained accuracy $=0.822$, sensitivity $=0.902$, specificity $=0$ of predicting MIC based on CA-125 concentration. No significant dependence of CA-125 and MIC was found. No relationship between disrupting factors and the presence of at least one LN with MIC without MAC per patient was found. 
Table 1. Study population overview.

\begin{tabular}{|c|c|c|}
\hline Variable & $\begin{array}{c}\text { Number of patients } \\
(\%)\end{array}$ & $\begin{array}{l}\text { Relationship to } \\
\text { CA-125 }\end{array}$ \\
\hline $\begin{array}{l}\text { CA-125 } \\
(4.4-137.5 ; \text { mean } 20.9)\end{array}$ & $45(100)$ & -- \\
\hline FIGO stage e $^{-1}$ & & $\mathrm{p}=0.467$ \\
\hline IA1 & $2(4.4)$ & \\
\hline IA 2 & $3(6.7)$ & \\
\hline IB1 & $37(82.3)$ & \\
\hline IB2 & $1(2.2)$ & \\
\hline IIA1 & $1(2.2)$ & \\
\hline IIA2 & $1(2.2)$ & \\
\hline Tumor histology & & $\mathrm{p}=0.907$ \\
\hline $\mathrm{SCC}$ & $39(86.6)$ & \\
\hline Adenocarcinoma & $3(6.7)$ & \\
\hline Other & $3(6.7)$ & \\
\hline Grade & & $\mathrm{p}=0.169$ \\
\hline 1 & $7(15.6)$ & \\
\hline 2 & $25(55.5)$ & \\
\hline 3 & $13(28.9)$ & \\
\hline Age & & $\mathrm{p}=0.045$ \\
\hline Premenopausal & 19 & \\
\hline Postmenopausal & 26 & \\
\hline Prior conization or amputation of the cervix & $10(22.2)$ & $\mathrm{p}=0.199$ \\
\hline Ovarian cyst & $1(2.2)$ & NA \\
\hline Endometriosis & $1(2.2)$ & NA \\
\hline Colon disease & $1(2.2)$ & NA \\
\hline Coexisting other cancer & $0(0)$ & -- \\
\hline Liver disease & $1(2.2)$ & NA \\
\hline LNM & $12(24)$ & $\mathrm{p}=0.287$ \\
\hline MAC/MIC & $8(17.7)$ & \\
\hline MIC without MAC & $4(8.8)$ & \\
\hline MIC & $4(8.8)$ & $\mathrm{p}=0.873$ \\
\hline Isolated MIC & $2(4.4)$ & \\
\hline MIC with ITC & $2(4.4)$ & \\
\hline LVLND (MIC and/or ITC) & $6(13.3)$ & $\mathrm{p}=0.423$ \\
\hline Isolated ITC & $4(8.8)$ & \\
\hline
\end{tabular}




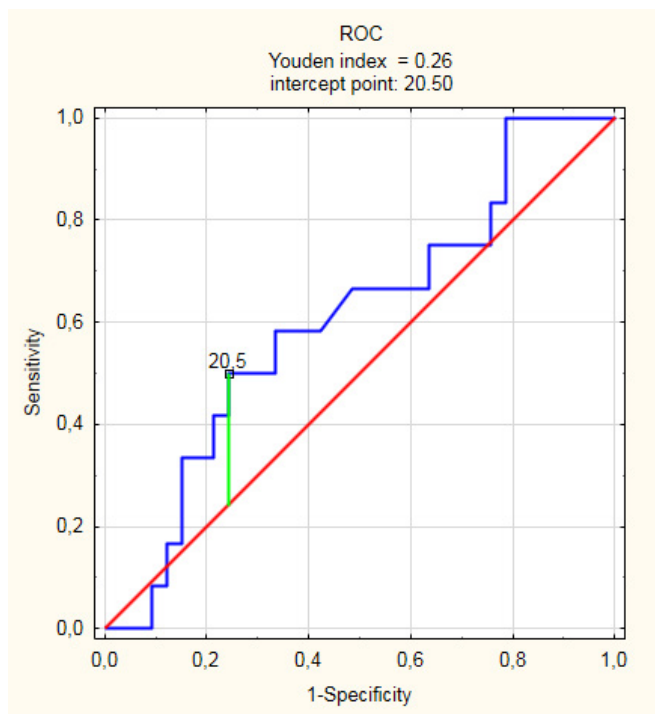

Figure 2. ROC curve for CA125 as a marker of LNM.

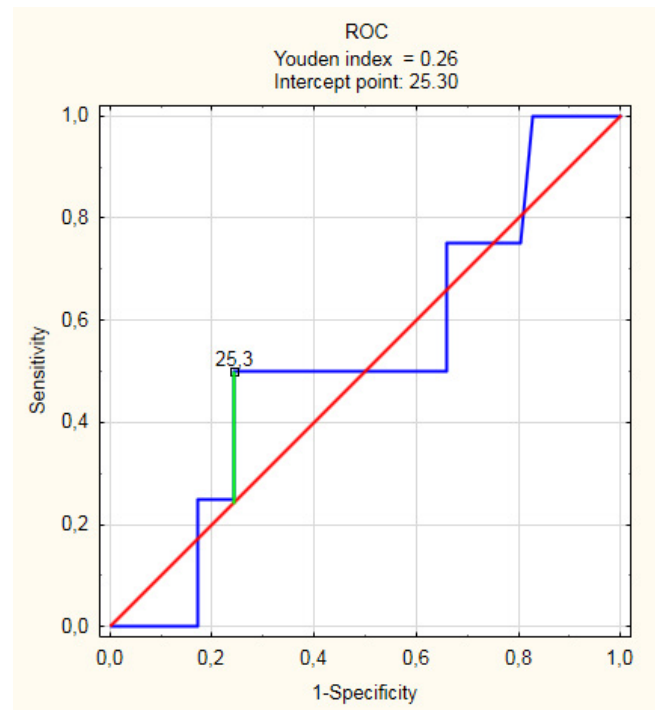

Figure 3. ROC curve for CA125 as a marker of MIC.

Table 2. Relationship between age and lymph node status.

\begin{tabular}{lllcc}
\hline Age & LNs negativity & LNM & $\begin{array}{c}\text { LVLND } \\
\text { positivity }\end{array}$ & Total \\
\hline Premenopausal & 9 & 12 & 5 & 18 \\
\hline Postmenopausal & 20 & 21 & 1 & 27 \\
\hline Total & 29 & 33 & 6 & 45 \\
\hline
\end{tabular}

LNs - lymph nodes; LNM - lymph node metastasis, LVLND - low volume lymph node disease;

Chi-square test: Age/LNs negativity, $p=0.041$, Age/LNM $p=0.187$, Age/LVLND $p=0.0285$

There was no statistically significant correlation between CA-125 and LVLND (M-W test, $\mathrm{p}=0.423$ ). The ROC curve does not statistically differ to the reference line (Figure 4). However, this trend is not statistically significant (AUC $=0.395 ; 95 \%$ CI $0.135-0.655, \mathrm{p}=0.4302$ ). For the cut-off point $35 \mathrm{U} / \mathrm{mL}$ we obtained accuracy $=0.80$, sensitivity $=0.167$, and specificity $=$ 0.897 in predicting LVLND based on CA-125 concentration.

\section{Discussion}

Ultrastaging is not routinely used in assessing LNM and causes stage migration, and consequently may provoke discrepancies between FIGO staging and actual prognosis. Previous studies did not focus on the relationship of biomarkers and low volume metastases, due to the fact that there was no accurate method for their preoperative analysis. It is important to ask whether the inexpensive and widely 


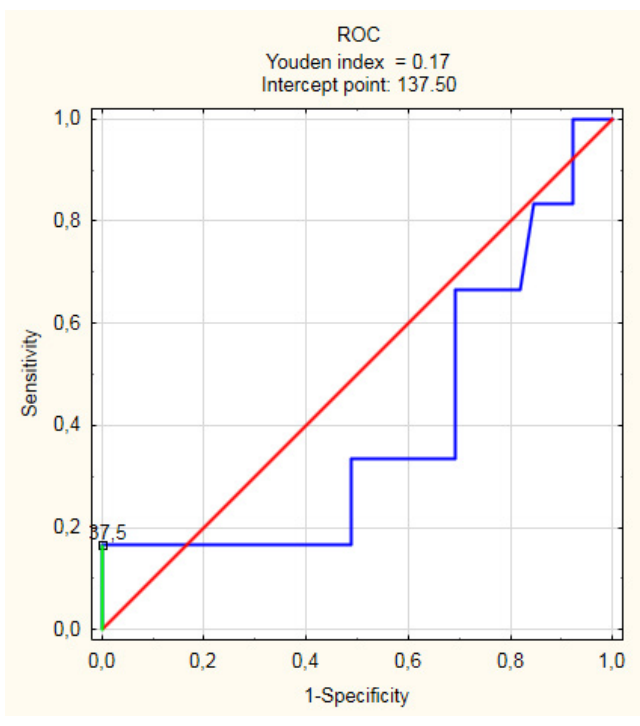

Figure 4. ROC curve for CA125 as a marker
of LVLND.

available CA-125 biomarker may be used for determination of MIC and ITC in patients with CC diagnosed before surgery. This could significantly help in targeting treatment as an element of tailoring therapy.

The study has several limitations. The first of them is that the number of patients was relatively small, and therefore the same is true of lymph node events. This is due to the fact that histopathological examination by means of histopathological ultrastaging is costly and time consuming and, therefore, difficult to perform routinely. The second is that there is no comparative research and also no previous own study available to validate our investigation. The third limitation is the use of a single tumor biomarker, which may be less specific in diagnosing target disease.

In both clinical experience and cited research, an elevated level of CA-125 was found in the poor outcome $\mathrm{CC}$ patients, especially where distant recurrences to LNs occur [24].
On the other hand, the correlation of CA-125 level with initial stage of disease cannot always be found [18, 24], however, it may be taken under consideration in determining the risk of parametrial infiltration [18]. Although the biology of CA-125 did not support the rationale of early detection of MIC to LN by CA-125, it is unknown whether CA-125 may indicate an altered state of health, caused by LVLND. In this study, the routine preoperative application of CA-125 was addressed to ultrastaged LNs. We found no correlation between preoperative concentration of CA-125 and LVLND. Also, no influence of this biomarker on the LN metastasis was found. However, it may be possible that at high concentrations of CA-125, diagnosis of LVLND may be less probable, as suggested by the ROC curve (below the reference line) in CA-125 and LVLND dependence analysis, and, in turn, it will be more probable to find MACs. Perhaps different levels of CA-125 identifying increased risk of LVLND in CC are different than those that help detect ovarian cancer. The premenopausal age was linked with LVLND, which may be explained by a long time of disease development - CC of lower biological stage at the time of diagnosis may be found in younger women. Nevertheless, both latter statements require evaluation on a larger group of patients.

CA-125 has a wide use in oncology. In the ovarian adenocarcinomas an elevated level of CA-125 after the treatment may indicate recurrence of disease in $2-8$ months before clinical manifestation [6]. On the other hand, Divine et al. showed that CA-125 $<81 \mathrm{U} / \mathrm{mL}$ at brain metastasis diagnosis in ovarian cancer is associated with longer survival [25]. In CC, CA125 is currently useful in pre-treatment prediction of prognosis, in particular in adenocarcinoma, and in prediction of LNM in this histological type as well. These applications have a level of evidence III-IV according to the Evidence Based Medicine principles [26]. The current 
study confirms this evidence. Therefore, it seems reasonable to test CA-125 in adenocarcinoma cases, especially in young women, who are at risk of ovarian recurrence when ovaries are preserved. In this study, this group was small ( 2 out of 3 patients with adenocarcinoma aged 25 and 30 had the ovary or ovaries left). To date no similar research has been done on $\mathrm{CC}$, and it is not known if CA-125 may serve as a predictor of recurrence in ovary-sparing cases. Nowadays, it becomes less routinely to perform imaging studies to monitor the treatment course of patients with cancer [27], and this is where the cancer biomarkers could be successfully implemented. However, when a method does not work at the beginning of a disease, it does not mean it will not support its monitoring or treatment of disease consequences. Hoogendam et al. stated that in the case of SCC relapses, SCC-Ag and high sensitive C-reactive protein (hsCRP) are the best biomarkers [28]. In our study no recurrence, but a risk of metastases to the lymph nodes, in any form - ITC, MIC or MAC, was assessed. We obtained high levels of

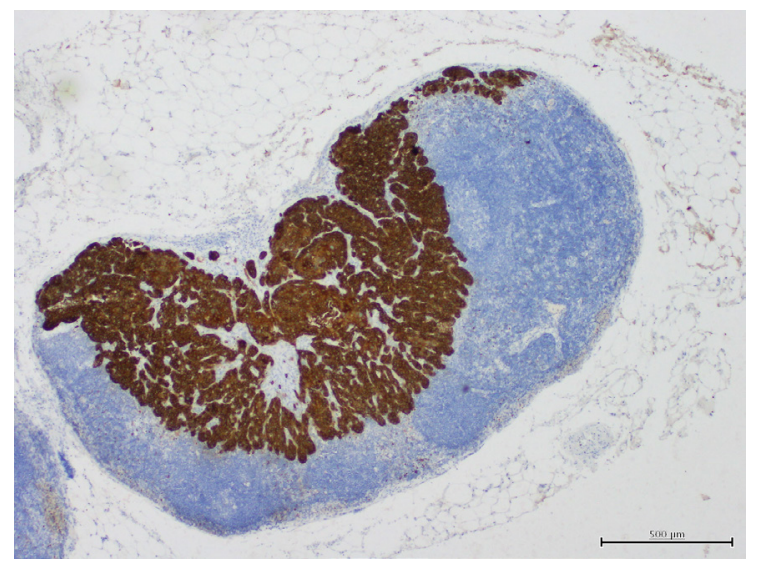

\footnotetext{
Supplemental material S1 Lymph node containing a micrometastasis of about 1.6 $\mathrm{mm}$ in greatest dimension - Magnification 40x, staining with immunohistochemistry (AE 1/3 antibodies).
}

specificity, and therefore the hypothesis about the usefulness of CA-125 in the range above the pathological values, in respect of the LNM, and LVLND prediction, may be rejected. The lower values (valid ones) are present in the majority of healthy women, who are not during menstruation or ovulation, therefore it is difficult to assume that they may have SCC, or may develop it in the future.

There was no statistically significant correlation of CA-125 concentration with the severity of the disease according to FIGO. However, it was eventually confirmed with ultrastaging. The fact that mean result of the CA-125 measurements was within the reference values confirms the limited usefulness of CA125 in predicting hidden metastatic diseases. The non-significant trend found between CA125 and LVLND could be just the effect of a small sample size (only 6 cases with LVLD). Perhaps at this moment, the inverse relationship can be explained by the fact that CA-125 can be considered as a marker of general cancer, hence its lower concentrations occur in cases of more advanced disease (not in case of LVLND). In future research, the relation between CA-125 and LVLND should be analyzed on a larger sample to confirm or reject the correlation between CA125 and LVLND. The employment of the nonspecific CA-125 biomarker was dictated by its low price and wide availability. Also, its nonspecificity was reduced by taking into account factors which could decrease its importance in CC. In some studies, in order to predict events such as parametrial or lymph node invasion, SCC-Ag is combined with CA-125. SCC marker was not used in our study due to economic reasons (health economic policy, institution economy), moreover it has no screening significance, and therefore it is not performed routinely in our center. The study by the Canadian Society of Gynecologic Oncology of Canada showed that 
operators expect a result of CA-125 only in the case of pelvic adnexal mass [29]. However, as showing the immunohistochemical study by Togami et al., CA-125 is strongly expressed by cancer cells of serous adenocarcinoma of the uterine cervix and mucinous endocervical adenocarcinoma [30]. However, they are less common than EOC.

To our knowledge, this is the first study evaluating a potential relationship between a serologic tumor marker (CA-125) and the LN metastatic disease, as detected by histopathological ultrastaging technique, in patients with early stages of CC.

Although CA-125 alone seems to be insufficient to assess the risk of finding LVLND in SCC of the cervix, perhaps together with SCC-Ag biomarker it would be a better predictor for LVLND. Moreover, an analysis of a larger group of patients with more varying disease severity status could enable finding the correlation between CA-125 and the early LNM status in some, more specified cases. This study, irrespective of negative results, brings new insight into the use of CA-125 in cervical cancer patients. According to the authors, the subject of LVLND should be given more consideration due to the fact that finding new uses for "old" biomarkers is much cheaper than developing new biomarkers.

\section{Acknowledgments}

The research was funded by the Ministry of Science and Higher Education allocated to maintain research potential from the statutory activities (GUMED ST02-0074/07 in years 2013-2015).

The study was approved by the Commission of Bioethics of the Medical University of Gdańsk, as a subanalysis from the main author's doctoral thesis (Ref. No. NKBBN/321/2013, approval date 15.07.2013).

\section{References}

1. Bray F, Jemal A, Grey N, Ferlay J, Forman D. Global cancer transitions according to the Human Development Index (2008-2030): a population-based study. Lancet Oncol. 2012 Aug;13(8):790-801. DOI: 10.1016/S14702045(12)70211-5

2. Reports based on the data the Oncology Centre: http://85.128.14.124/krn/, www.onkologia.org. Access: 10.12.2017 05.04.2017.

3. Høgdall EV, Christensen L, Kjaer SK, Blaakaer J, Kjaerbye-Thygesen A, Gayther S, et al. CA125 expression pattern, prognosis and correlation with serum CA125 in ovarian tumor patients. From The Danish "MALOVA" Ovarian Cancer Study. Gynecol Oncol. 2007 Mar;104(3):508-15. DOI: 10.1016/j.ygyno.2006.09.028

4. Scharl A, Crombach G, Vierbuchen M, Müsch H, Bolte A. CA 125 in normal tissues and carcinomas of the uterine cervix, endometrium and fallopian tube. I. Immunohistochemical detection. Arch Gynecol Obstet. 1989 Jun;244(2):103-12. DOI: 10.1007/BF00931381 
5. Sadowski EA, Robbins JB, Guite K, Patel-Lippmann K, Del Rio AM, Kushner DM, et al. Preoperative pelvic MRI and serum cancer antigen-125: selecting women with grade 1 endometrial cancer for lymphadenectomy. AJR Am J Roentgenol. 2015 Nov;205(5):W556-64. DOI: $10.2214 /$ AJR.14.13746

6. Rizzuto I, Stavraka C, Chatteriee J, Borley J, Hopkins TG, Gabra H, et al. Risk of Ovarian Cancer Relapse Score: a prognostic algorithm to predict relapse following treatment for advanced ovarian cancer. Int J Gynecol Cancer. 2015 Mar;25(3):416-22. DOI: 10.1097/IGC.0000000000000361

7. Tsai CC, Liu YS, Huang EY, Huang SC, Chang HW, Tseng CW, ChangChien CC et al. Value of preoperative serum CA125 in early-stage adenocarcinoma of the uterine cervix without pelvic lymph node metastasis. Gynecol Oncol. 2006 Mar;100(3):591-5. DOI: 10.1016/j.ygyno.2005.09.049

8. Gadducci A, Tana R, Cosio S, Genazzani AR. The serum assay of tumour markers in the prognostic evaluation, treatment monitoring and follow-up of patients with cervical cancer: a review of the literature. Crit Rev Oncol Hematol. 2008 Apr;66(1):10-20. DOI: 10.1016/j. critrevonc.2007.09.002

9. Porika M, Vemunoori AK, Tippani R, Mohammad A, Bollam SR, Abbagani S. Squamous cell carcinoma antigen and cancer antigen 125 in southern Indian cervical cancer patients. Asian Pac J Cancer Prev. 2010 Jun;11(6):1745-7.

10. Takeda M, Sakuragi N, Okamoto K, Todo Y, Minobe S, Nomura E, et al. Preoperative serum SCC, CA125, and CA19-9 levels and lymph node status in squamous cell carcinoma of the uterine cervix. Acta Obstet Gynecol Scand. 2002 May;81(5):451-7. DOI: 10.1034/j.16000412.2002.810513.x

11. Koper NP, Thomas CM, Dorn KE, Schijf CP. Improvement of clinical staging in cervical cancer with serum squamous cell carcinoma antigen and CA 125 determinations. Gynecol Oncol. 1997 Mar;64(3):473-6. DOI: $10.1006 /$ gyno.1996.4581

12. Avall-Lundqvist EH, Sjövall K, Nilsson BR, Eneroth $\mathrm{PH}$. Prognostic significance of pretreatment serum levels of squamous cell carcinoma antigen and CA 125 in cervical carcinoma. Eur J Cancer. 1992;28A(10):1695702. DOI: 10.1016/0959-8049(92)90071-9
13. Davelaar EM, van de Lande J, von Mensdorff-Pouilly $\mathrm{S}$, Blankenstein MA, Verheijen RH, Kenemans P. A combination of serum tumor markers identifies high-risk patients with early-stage squamous cervical cancer. Tumour Biol. 2008;29(1):9-17. DOI: $10.1159 / 000132566$

14. Ueda Y, Enomoto T, Kimura T, Miyatake T, Yoshiho $\mathrm{K}$, Fujita M, et al. Serum biomarkers for early detection of gynecologic cancers. Cancers (Basel). 2010 Jun 14;2(2):1312-27. DOI: $10.3390 /$ cancers2021312

15. Bats A, Mathevet P, Buenerd A, Orliaquet I, Mery E, Zerdoud S, et al. The sentinel node technique detects unexpected drainage pathways and allows nodal ultrastaging in early cervical cancer: insights from the multicenter prospective SENTICOL study. Ann Surg Oncol. 2013 Feb;20(2):413-22. DOI: 10.1245/s10434012-2597-7

16. Borras G, Molina R, Xercevanis J, Ballesta A, Iglesias J. Tumor antigens CA 19.9, CA 125, and CEA in carcinoma of the uterine cervix. Gynecol Oncol. 1995 May;57(2):205-11. DOI: 10.1006/gyno.1995.1126

17. Kotowicz B, Kaminska J, Fuksiewicz M, Kowalska M, Jonska-Gmyrek J, Gawrychowski K, et al. Clinical significance of serum CA-125 and soluble tumor necrosis factor receptor type I in cervical adenocarcinoma patients. Int J Gynecol Cancer. 2010 May;20(4):588-92. DOI: $10.1111 /$ IGC.0b013e3181d5c27a

18. Yamazaki H, Todo Y, Okamoto K, Yamashiro K, Kato H. Pretreatment risk factors for parametrial involvement in FIGO stage IB1 cervical cancer. J Gynecol Oncol. 2015 Oct;26(4):255-61. DOI: 10.3802/jgo.2015.26.4.255

19. Darai E, Rouzier R, Ballester M, Barranger E, Coutant C. Sentinel lymph node biopsy in gynaecological cancers: the importance of micrometastases in cervical cancer. Surg Oncol. 2008 Sep;17(3):227-35. DOI: 10.1016/j.suronc.2008.04.002

20. Slama J, Dundr P, Dusek L, Fischerova D, Pinakavova I, Vrzackova P, et al. Sentinel lymph node status in patients with locally advanced cervical cancers and impact on neoadjuvant chemotherapy. Gynecol Oncol. 2012 May;125(2):303-6. DOI: 10.1016/j.ygyno.2012.02.010

21. Pecorelli S. Revised FIGO staging for carcinoma of the vulva, cervix, and endometrium. Int $\mathrm{J}$ Gynaecol Obstet. 2009 May;105(2):103-4. DOI: 10.1016/j. ijgo.2009.02.009 DOI: 10.1016/j.ijgo.2009.02.012 
22. Schwartz GF, Giuliano AE, Veronesi U. Proceedings of the consensus conference on the role of sentinel lymph node biopsy in carcinoma of the breast. April 15-22, 2001. Philadelphia. Pennsylvania. Cancer. 2002 May 15;94(10):2542-51. DOI: 10.1002/cncr.10539

23. Singletary SE, Greene FL. Revision of breast cancer staging: the 6th edition of the TNM Classification. Semin Surg Oncol. 2003 Nov;21(1):53-9. DOI: $10.1002 /$ ssu. 10021

24. Goldberg GL, Sklar A, O'Hanlan KA, Levine PA, Runowicz CD. CA-125: a potential prognostic indicator in patients with cervical cancer? Gynecol Oncol. 1991 Mar;40(3):222-4. DOI: 10.1016/0090-8258(90)90281$\mathrm{O}$

25. Divine LM, Kizer NT, Hagemann AR, Pittman ME, Chen L, Powell MA, et al. Clinicopathologic characteristics and survival of patients with gynecologic malignancies metastatic to the brain. Gynecol Oncol. 2016 Jul;142(1):76-82. DOI: 10.1016/j.ygyno.2016.04.030

26. Dasari S, Wudayagiri R, Valluru L. Cervical cancer: biomarkers for diagnosis and treatment. Clin Chim Acta. 2015 May 20;445:7-11. DOI: $10.1016 /$ j. cca.2015.03.005
27. Rimel BJ, Burke WM, Higgins RV, Lee PS, Lutman $\mathrm{CV}$, Parker L. Improving quality and decreasing cost in gynecologic oncology care. Society of gynecologic oncology recommendations for clinical practice. Gynecol Oncol. 2015 May;137(2):280-4. DOI: 10.1016/j.ygyno.2015.02.021

28. Hoogendam JP, Zaal A, Rutten EG, Heijnen CJ, Kenter GG, Veldhuis WB, et al. Detection of cervical cancer recurrence during follow-up: a multivariable comparison of 9 frequently investigated serum biomarkers. Gynecol Oncol. 2013 Dec;131(3):655-60. DOI: 10.1016/j.ygyno.2013.10.016

29. Kupets R, Giede KC, Power P, Agrawal A; Executive of the Society Gynecologic Oncology of Canada. The investigations required before referring a patient to a gynecologic oncologist. J Obstet Gynaecol Can. 2016 Feb;38(2):164-7. DOI: 10.1016/j.jogc.2015.12.016

30. Togami S, Sasajima Y, Kasamatsu T, Oda-Otomo R, Okada S, Ishikawa $\mathrm{M}$, et al. Immunophenotype and human papillomavirus status of serous adenocarcinoma of the uterine cervix. Pathol Oncol Res. 2015 Apr;21(2):487-94. DOI: 10.1007/s12253-014-9854-y 